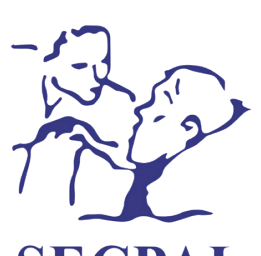

SECPAL

\title{
Diplopia en un paciente con cáncer de próstata avanzado
}

\author{
Álvaro Sanz ${ }^{* 1}$, Alba Puente $^{1}$, Gabriel Carlos Fernández ${ }^{2}$ y María Luisa del Valle ${ }^{3}$
}

'Sección de Oncología Médica, Hospital Universitario del Río Hortega, Valladolid. ${ }^{2}$ Servicio de Radiodiagnóstico, Hospital
Universitario del Río Hortega, Valladolid. ${ }^{3}$ Servicio de Radioterapia, Hospital Clínico Universitario de Valladolid. España

Recibido el 1 de febrero de 2018

Aceptado el 14 de mayo de 2018

Sanz A, Puente A, Fernández GC, del Valle ML. Diplopia en un paciente con cáncer de próstata avanzado. Med Paliat. 2019;26(1):84-86.

\begin{abstract}
Varón de 75 años, diagnosticado en 2005 de carcinoma de próstata avanzado con evidencia de metástasis óseas múltiples. Fue tratado inicialmente con bloqueo androgénico (goserelina y bicalutamida), con lo que alcanzó respuesta con normalización de los niveles de PSA. Cuando se detectó progresión se retiró el antiandrógeno (bicalutamida). En 2015 recibió radioterapia paliativa lumbar y comenzó tratamiento con abiraterona y prednisona, con estabilización transitoria de la enfermedad. En marzo de 2016 se evidenció progresión, pero el paciente descartó recibir quimioterapia. Se administró de nuevo radioterapia paliativa, en esta ocasión dorsal, se retiró entonces la abiraterona y se mantuvo la prednisona, lo que se asoció a un descenso inesperado y mantenido de PSA.

En septiembre de 2017 fue valorado en Urgencias por padecer diplopia sin otras manifestaciones neurológicas. Se le realizó una TAC craneal.

La TAC craneal evidenció afectación ósea de la base del cráneo, el clivus y el ala mayor del esfenoides derecho extendida a la pared lateral de la órbita con afectación de partes blandas (Figura 1) y exoftalmos secundario (Figura 2). Estos datos justificaban la clínica de diplopia. Como hallazgo inesperado se encontró un hematoma subdural frontoparietal derecho que alcanzaba un grosor de 1,5 cm (Figura 3) y
\end{abstract}

que provocaba desplazamiento de la línea media y compresión del sistema ventricular adyacente (Figura 4). El diagnóstico fue de hematoma subdural subagudo en el contexto de carcinoma de próstata metastásico con diseminación en huesos del cráneo y probable afectación dural.

Tres días más tarde ingresó por episodios de pérdida de conocimiento y relajación de esfínteres. En una nueva TAC craneal no se encontró evidencia de nuevo sangrado. La situación neurológica se fue deteriorando y falleció tras cuatro días del ingreso.

Desde una perspectiva didáctica se podría decir que las metástasis en la duramadre (la capa meníngea más externa y adherida a la pared craneal) se encuentran en una situación intermedia y comparten características de las metástasis en los huesos craneales y de la diseminación leptomeníngea clásica, la carcinomatosis meníngea1. Esta afectación dural puede presentarse tanto por diseminación hematógena meníngea como por progresión local desde los huesos craneales e incluso desde metástasis cerebrales ${ }^{2}$. Si en las necropsias de pacientes con tumores sólidos avanzados se describe una incidencia cercana al 20 \% de metástasis leptomeníngeas ${ }^{3}$, la probabilidad de encontrar afectación dural es menor, por debajo del $10 \%{ }^{4}$. Y si el cáncer de mama es el tumor que se encuentra con más frecuencia en caso de

\footnotetext{
*Autor para correspondencia:

Álvaro Sanz Rubiales

Oncología Médica. Hospital Universitario del Río Hortega. c/ Dulzaina, 1. 47012, Valladolid, España

Correo electrónico: asrubiales@hotmail.com
} 


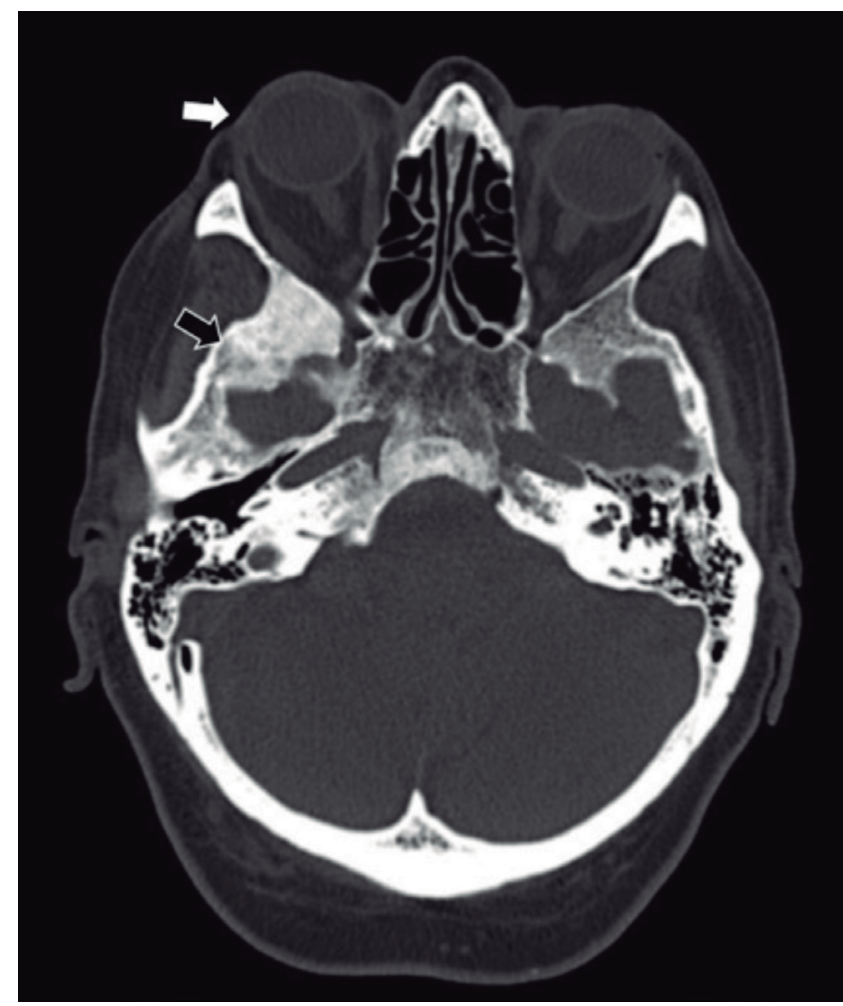

Figura 1. Afectación ósea de aspecto blástico del ala mayor del esfenoides derecho extendida a la pared lateral de la órbita (flecha negra) y exoftalmos secundario (flecha blanca).

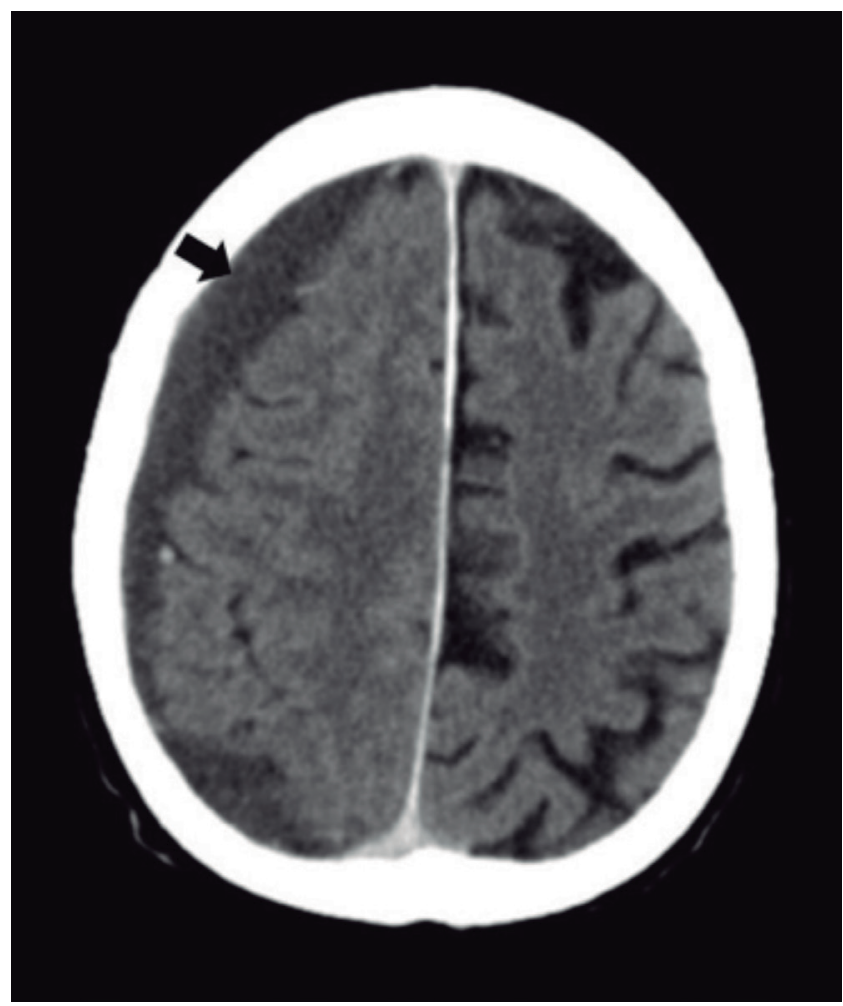

Figura 3. TAC craneal: hematoma subdural frontoparietal derecho (flecha).

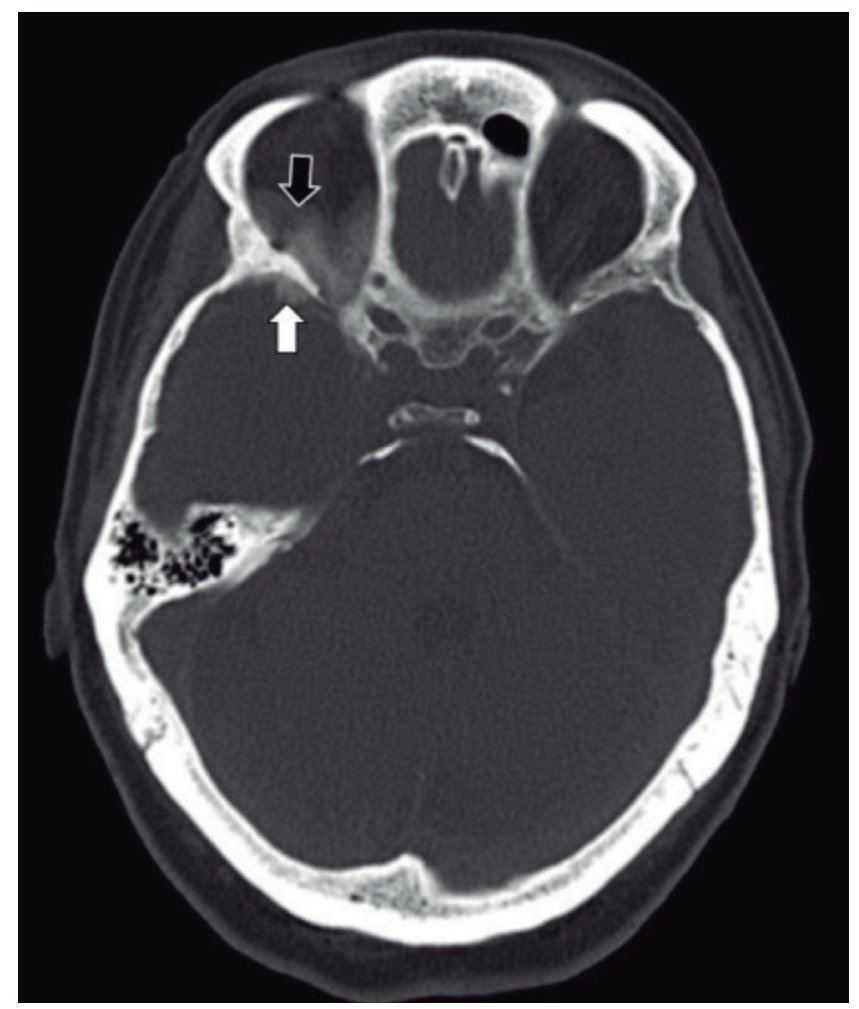

Figura 2. Infiltración de partes blandas de la región posterior de la órbita derecha (flecha negra) con una probable infiltración dural (flecha blanca).

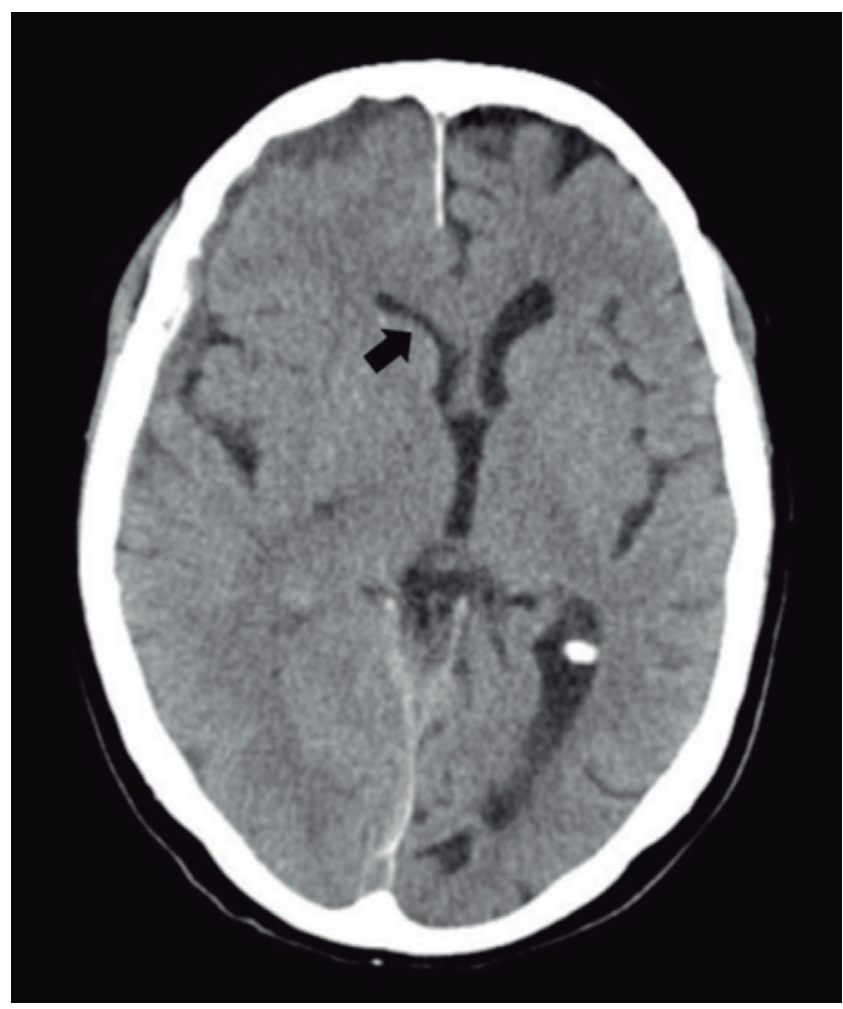

Figura 4. Desplazamiento de la línea media y compresión del sistema ventricular (flecha) secundario al efecto masa producido por el hematoma subdural. 
diseminación leptomeníngea ${ }^{3}$, en la afectación en duramadre es el de próstata ${ }^{4}$. La metástasis dural es asintomática en la mayor parte de los casos, ya que solo se describe una incidencia clínica cercana al $1 \%$ en cáncer de próstata avanzado ${ }^{5}$. Los síntomas pueden deberse a la afectación del hueso subyacente o a manifestaciones neurológicas por infiltración meníngea o por sangrado subdural no traumático, un fenómeno descrito desde hace más de cien años ${ }^{6}$. La probabilidad de que las metástasis durales provoquen un hematoma subdural (por sangrado del tumor y/o infiltración de la trama venosa de la duramadre ${ }^{7}$ ) es muy escasa ${ }^{6,8}$ y se ha estimado en torno al 0,1\% en hematomas crónicos ${ }^{2}$. En ocasiones puede haber dificultades diagnósticas, ya que los estudios de imagen no siempre permiten discriminar entre sangrado y metástasis dural ${ }^{9}$.

La actuación clínica en pacientes con hematoma subdural secundario a metástasis dural debe adaptarse a la situación clínica, la expectativa de mejoría y la diseminación, evolución y posibilidades de tratamiento de la neoplasia de base ${ }^{10}$. Cuando es factible, se plantea un drenaje quirúrgico del hematoma para administrar secundariamente radioterapia y/o tratamiento sistémico de las metástasis. En pacientes con mayor deterioro hay que ser prudente en la toma de decisiones, ya que a la situación de cáncer avanzado y al daño neurológico debido al hematoma se pueden añadir las complicaciones quirúrgicas o postquirúrgicas (locales o sistémicas) y el riesgo de un nuevo sangrado, sobre todo si se asocian alteraciones de la coagulación².

\section{Bibliografía}

1. Rodríguez Mowbray JR, Macías Montero R, Gómez-Ulla Astray J. Carcinomatosis meníngea: distintos casos con los mismos resultados. MEDIPAL. 2014;21(3):121-5.

2. George KJ, Lau A, Ellis M, Kiehl TR, Fehlings MG. Metastatic coagulopathic subdural hematoma: A dismal prognosis. Surg Neurol Int. 2012;3:60.

3. Chamberlain MC. Neoplastic meningitis. Oncologist. 2008;13(9):967-77.

4. Laigle-Donadey F, Taillibert S, Mokhtari K, Hildebrand J, Delattre JY. Dural metastases. J Neurooncol. 2005;75(1):57-61.

5. Benjamin R. Neurologic complications of prostate cancer. Am Fam Physician. 2002;65:1834-40.

6. Kunii N, Yoshikawa G, Kirino T. Subdural hematoma associated with dural metastasis -case report-. Neurol Med Chir (Tokyo). 2005;45(10):519-22.

7. Boukas A, Sunderland GJ, Ross N. Prostate dural metastasis presenting as chronic subdural hematoma. A case report and review of the literature. Surg Neurol Int. 2015;6:30.

8. Cobo Dols M, Gil Calle S, Villar Chamorro E, Ales Díaz I, Montesa Pino A, Alcaide García J, et al. Dural metastases with subdural hematoma from prostate cancer. Oncología. 2005;28:407-11.

9. Nzokou A, Magro E, Guilbert F, Fournier JY, Bojanowski MW. Subdural metastasis of prostate cancer. J Neurol Surg Rep. 2015;76(1):e123-7.

10. Reichman J, Singer S, Navi B, Reiner A, Panageas K, Gutin PH, et al. Subdural hematoma in patients with cancer. Neurosurgery. 2012;71(1):74-9. 